\title{
Factors Affecting Specific Rehabilitation in Follow-Up of Patients Coming to Outpatient Physiotherapy Department: An Online Social Networking (OSN) Survey
}

Dr. Trupti S. Kulkarni Deshmukh ${ }^{1 *}$, Dr. Pooja Agarwala Mahajan², Dr. Shweta Satish Devare Phadke ${ }^{3}$

\footnotetext{
${ }^{1,2}$ Assistant professor at TMV's Lokmanya Medical college of Physiotherapy, Navi Mumbai India

${ }^{3}$ Professor \& Principal, at TMV's Lokmanya Medical college of Physiotherapy, Navi Mumbai India
}

DOI: $10.36347 /$ sjams.2020.v08i05.024

| Received: 07.05.2020 | Accepted: 14.05.2020 | Published: 17.05.2020

*Corresponding author: Dr. Trupti S. Kulkarni Deshmukh

\section{Abstract}

Discontinuation of follow up is common in patients of physiotherapy. Factors for discontinuation may be specific for specific rehabilitation like Orthopedic, Neurology, sports, community and cardio-respiratory physiotherapy. Many studies focused on common factors for discontinuation. This study aim at assessing factors affecting specific rehabilitation follow up of patients coming to OPD of physiotherapy department using online social network. This is a cross sectional survey through online social network (OSN) carried out on 500 patients who discontinued coming to physiotherapy OPD for 6 month duration. Pre validated questionnaire was used to collect data of patients who discontinued physiotherapy. Data was collected and patients were categorized in Ortho, neuro, community, cardiorespiratory, sports rehabilitation follow up. Post study percentages of factors affecting specific rehabilitation follow up were calculated by statistical analysis. By the end of the study, unavailability of modality (56\%), Long waiting time (31\%),timings not suitable to patient's schedule(13\%)were factors for ortho, Long waiting time $(60 \%)$, Patient not finding the need for physiotherapy(22\%), unavailability of caregivers(18\%) to take to hospital for Neuroreahabilitation, High cost of treatment $(88 \%)$ for sports rehabilitation, Doctor hasn't advised (56\%) and timings not suitable to schedule (44\%) for community rehabilitation. Therapist not advised (44\%) and high cost (56\%)were some of the factors affecting follow up in cardio-respiratory rehabilitation.Physiotherapy department should approach every patient not coming for follow up through online social networking site on regular basis post OPD visit. Therapist should take necessary steps to reduce patients waiting time, give appointment considering patients availability, give manual therapy over modality, refer patients to psychologist and also conduct awareness camps for field specific rehabilitation in patients, caregivers and other healthcare workers in order to maintain the patient follow-up.

Keywords: Follow-up, Outpatient Department, Rehabilitation, Online social networking (OSN), Appointment.

Copyright @ 2020: This is an open-access article distributed under the terms of the Creative Commons Attribution license which permits unrestricted use, distribution, and reproduction in any medium for non-commercial use (NonCommercial, or CC-BY-NC) provided the original author and source are credited.

\section{INTRODUCTION}

Physiotherapy department is one of the very potential departments in hospital setup. Follow up is next visit to the department to improve treatment on the basis of patient's improvement[1].

Follow up is very important factor in treatment of physiotherapy. Follow up helps physiotherapist to progress patient's treatment on the basis of specific protocol in further weeks or months depending upon patients diagnosis and makes him functionally independent.

Therapist advice depending on current status on patient, changes in previous visit prescribed exercises, changes in prescribed frequency of exercises, increasing complexity depending on rehabilitation protocol are important aims of physiotherapy follow-up visits[2].

As the pain decreases and patient achieves functional independence post primary rehabilitation it has been observed that there is ignorance in patient for follow up. High packages, long waiting time, quality of treatment, unavailability of modality $[3,4]$ are some of other factors responsible given in literature responsible for follow up ignorance in patients.

Now a day's all individuals use all social networking application and hence are easily accessible on whatsapp, facebook messengers, instagram etc [5]. In this study, all previous patients were contacted via social networking applications on the basis of their 
stored data in outpatient physiotherapy department and questionnaire were sent to them.

It has been observed that many patients are not coming for regular follow up in physiotherapy outpatients department and as a result of this; the number of patients coming to OPD is decreasing day by day.

Many studies only focused on reason of non adherence with physiotherapy rehabilitation [2, 3]. Few studies are available which focused on factors of discontinuing of follow up depending on specific specialization categories like ortho, Neuro, Community, Sports or Cardio-respiratory rehabilitation.

This study is conducted to find out factors which are causing the patients to discontinue with treatment so that specific department of physiotherapy can make necessary changes in quality of treatment. Feedback through social application will be helpful to store data and provide special follow up schemes to the patients. Study also helps to convert most of the new cases into regular follow up cases by improving services and solving common problems faced by patients to continue the treatment.

Study aim is to find out factors affecting patients for regular follow-up in specific rehabilitation outpatient physiotherapy department of multidisciplinary hospital.

\section{Methods And Methodology}

Study Design: Cross sectional survey through online social network (OSN).

Sampling Method: Purposive sampling.

Sample Size: 500 (OPD patients).

Study Duration: 6 months.

Study Setup: Outpatient physiotherapy department of multidisciplinary hospital.

Inclusion criteria: Patients coming in physiotherapy multidisciplinary department.

Exclusion criteria: IPD patients were excluded.

\section{Methodology}

In one multidisciplinary hospital pilot survey was conducted. On the basis of pilot survey Digital questionnaire were made in three regional languages (Marathi, Hindi, English). List of patients stored data of name and contact details of past 1 year were collected. Prior consent was taken from patient. Questionnaire was made on the basis of common problems faced by patients to continue with treatment. Questionnaire was forwarded to patients using different online social networking sites. Digital questionnaire were forwarded to 700 patients who were suppose to come for follow up for next level rehabilitation but did not come. Out of 700 only 500 patients filled the Digital questionnaire and Data was collected and patients were categorized into Ortho, neuro, community, cardio-respiratory and sports rehabilitation follow up and statistically analyzed.

\section{RESULTS AND DiSCUSSION}

Data Analysis

Table-1: Specific rehabilitation follows up patient's data

\begin{tabular}{|c|c|}
\hline Specific Rehabilitation & Percentage of patients \\
\hline Ortho-Rehabilitation & $64 \%$ \\
\hline Sports-Rehabilitation & $16 \%$ \\
\hline Neuro-Rehabilitation & $13 \%$ \\
\hline Community-Rehabilitation & $4 \%$ \\
\hline Cardio-Respiratory-Rehabilitation & $3 \%$ \\
\hline
\end{tabular}

1.SPECIFIC REHABILITATION FOLLOW UP PATIENT'S DATA

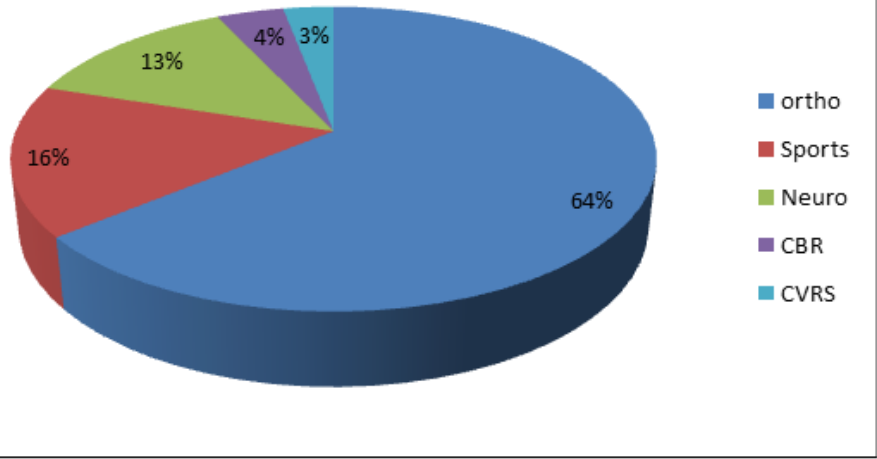


Table-2: Factors affecting specific reahabilitation data

\begin{tabular}{|c|c|c|c|c|c|c|c|c|c|c|c|}
\hline \multicolumn{3}{|c|}{ Ortho-Rehabilitation } & \multicolumn{2}{|c|}{$\begin{array}{l}\text { Sports- } \\
\text { Rehabilitation }\end{array}$} & \multicolumn{3}{|c|}{ Neuro-Rehabilitation } & \multicolumn{2}{|c|}{$\begin{array}{l}\text { Community- } \\
\text { Rehabilitation }\end{array}$} & \multicolumn{2}{|c|}{$\begin{array}{l}\text { Cardio- } \\
\text { Respiratory- } \\
\text { Rehabilitation }\end{array}$} \\
\hline $\begin{array}{l}\text { Unavailability } \\
\text { of modality }\end{array}$ & $\begin{array}{l}\text { Long } \\
\text { waiting } \\
\text { time }\end{array}$ & $\begin{array}{l}\text { Timing } \\
\text { not } \\
\text { suitable }\end{array}$ & $\begin{array}{l}\text { High } \\
\text { Cost }\end{array}$ & $\begin{array}{l}\text { Long } \\
\text { waiting } \\
\text { time }\end{array}$ & $\begin{array}{l}\text { Long } \\
\text { waiting } \\
\text { time }\end{array}$ & $\begin{array}{l}\text { Myself } \\
\text { not } \\
\text { required }\end{array}$ & $\begin{array}{l}\text { Unavailability } \\
\text { of caregivers }\end{array}$ & $\begin{array}{l}\text { Doctor } \\
\text { not } \\
\text { advised }\end{array}$ & $\begin{array}{l}\text { Timing } \\
\text { not } \\
\text { suitable }\end{array}$ & $\begin{array}{l}\text { Therapist } \\
\text { not } \\
\text { advised }\end{array}$ & $\begin{array}{l}\text { High } \\
\text { Cost }\end{array}$ \\
\hline $56 \%$ & $31 \%$ & $13 \%$ & $88 \%$ & $12 \%$ & $60 \%$ & $22 \%$ & $18 \%$ & $56 \%$ & $44 \%$ & $44 \%$ & $56 \%$ \\
\hline
\end{tabular}

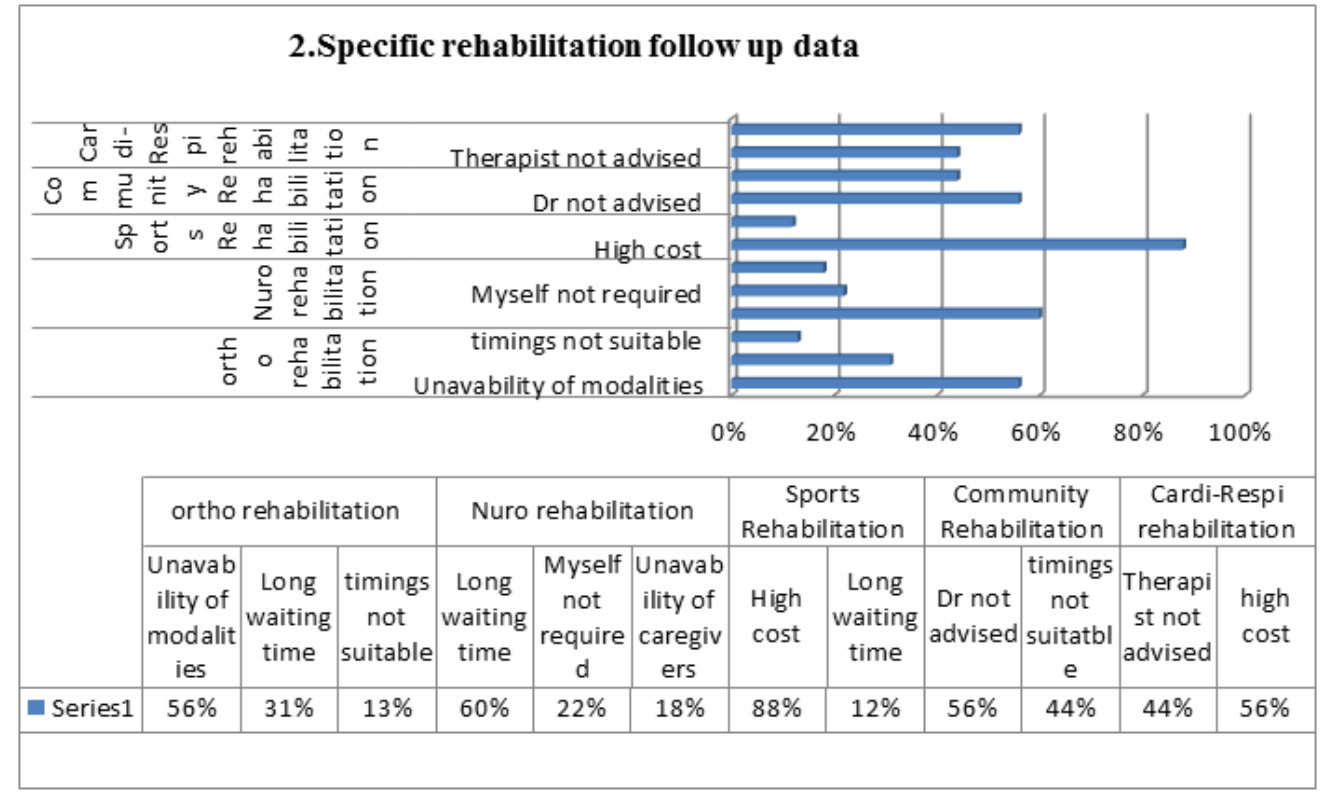

\section{DISCUSSION}

As per above data collected and analyzed it is observed that patients were from ortho 64\%, Sports $16 \%$ and Neuro 13\%,Community based rehabilitation $4 \%$ and Cardiovascular surgery rehabilitation(CVRS) $3 \%$.

Probable reason for discontinuing treatment observed in this study for ortho rehabilitation patient was unavailability of modalities $(56 \%)$ for longer time as well as timings were not suitable(13\%) to their daily schedule.

Ortho rehabilitation is weekly progressive rehabilitation and requires patient understanding about importance of therapy and need to take out time for the treatment.

Ortho rehabilitation also requires multiple modalities (modalities to reduce pain, to improve range of motion as well as modalities to improve strength and also for stretching).Hospital setups are not having enough number of machines to provide treatment to new as well as follow up patients at the same time and therefore increases waiting time of patients $(31 \%)$.

Follow up for sports rehabilitation was also a long process. Sports rehabilitation requires modalities which are very expensive and can be used only for one time. Kinesio tapping, dry Needling, cupping therapy are some of the examples of expensive sports rehabilitation materials[6]. In this study we also found out that sports follow up patient discontinue with the treatment due to high treatment charges $(88 \%)$.

Large numbers of neuro rehabilitation patients were also found out to discontinue treatment and when studied it was observed that most of them stay far away from the OPD set up and neuro patients required an assistant or a helper to carry them to the hospital $(18 \%)[7]$.

Most of the neuro patients also experience depression and poor quality of life [8] and by themselves decideto discontinue treatment(22\%)[9]. For these patients, the rehabilitation also requires long time and could be the reason for long waiting time $(60 \%)$.

In case of community rehabilitation follow up, it was found that most of the patients were not advised by Doctors $(56 \%)$. Awareness of physiotherapy in women's health[10],industrial rehabilitation programs $[11,12]$ as well as geriatric health rehabilitations [13, 14]were found less in doctors which in turn reduced the referral for physiotherapy. Patients also found that timings were not suitable, because most of the community rehabilitation services were taken by women who generally have household work in morning time, IT professionals are busy in office work and visiting the OPD was difficult for them at the cost of their jobs and the Geriatric population which needed a 
helper to bring them to the OPD was not readily availableat all times which caused a hindrance for their follow-up visits.

In case of Cardio-Respiratory rehabilitation most of the patients reported that the therapist advised them to do exercises at home $(44 \%)$. Prevention of hospital acquired infections to the patients could be the reason for therapist advice in this case. [15]Good hygiene for preventing infection to these patients requires high quality of sterilization. Modalities for rehabilitation of these patients are also expensive and require high maintenance cost and therefore the treatment charges for each session were found to be costly for follow up patients $(56 \%)$.

From this study it has been observed that hospital physiotherapy department should conduct awareness camps for doctors as well as therapists to emphasize on the need of follow ups. Follow ups are necessary for patients as well as for the hospital benefits. Counseling of patients at the time of discharge also plays an integral role for follow up. Psychological counseling of patients as well as relatives at the time of discharge especially in neuro patients will improve the follow up count of physiotherapy department. Increasing the number of modalities, assigning different suitable appointment time for follow up patients will reduce waiting time. In case of sports, therapist can ask patients to get their own material for rehabilitation for next follow up so that there will be cost cutting for the patients. Therapist can conduct small antenatal postnatal exercise demos for gynecologist so that they can refer more patients for follow ups. Group exercises can be conducted where the patient can pay a minimal amount which is affordable for the patient, mainly the geriatric and women's population. Ergonomic advice sessions can be conducted in industries to improve follow up. Infection prevention can be taken care before giving rehabilitation to cardio-respiratory follow up patients.

Clinical significance-This study will help the therapist to find out reason for discontinuing follow up using online social network and taking necessary steps in order to improve follow ups. Therapist can also share some good offers for follow ups.

\section{CONCLUSION}

This study concluded that factors affecting follow ups were different for the patients coming for different rehabilitation (ortho, neuro, sports, and community, cardio-respiratory).

Long waiting time, unavailability of modalities for longer time, high follow up charges, self avoidance due to psychological factors, staying away from treatment centers, unavailability of caregivers to carry patient to hospital, not advised by Doctors or sometimes by therapist were common factors found to be affecting follow up of patients.

Social Networking sites were found effective and easily accessible medium to reach patients. Therefore study concluded that, Physiotherapy department should approach every patient not coming for follow up through online social networking site on regular basis. Therapist should take necessary steps to reduce patients waiting time, giving appointment as per their suitable time, and giving more manual therapy over modality ,focusing more on group exercises and referring them to psychologist whenever necessary and also by conducting awareness camp for field specific rehabilitation in patients, caregivers and other healthcare workers.

\section{REFRENCES}

1. Kyriacou DN. Brief Report: Factors affecting outpatient follow-up compliance of emergency department patients. Journal of general internal medicine. 2005. 20(10): 938-942.

2. Jack K. Barriers to treatment adherence in physiotherapy outpatient clinics: a systematic review. Manual therapy. 2010; 15(3): 220-228.

3. Kanika, M, H. Horobin and S. McLean, Indian physiotherapists' perceptions of factors that influence the adherence of Indian patients to physiotherapy treatment recommendations. International Journal of Physiotherapy and Rehabilitation; 2010.

4. Olaleye O and K Suddick. A study of perceived factors affecting patients' participation in outpatient stroke physiotherapy exercise in Nigeria. International Journal of Therapy and Rehabilitation. 2012;19(10): 581-590.

5. Grover P, Deshmukh TK and Devare Phadke S. Efficacy of online social networking (osn) in creating awareness about cervical and shoulder related musculoskeletal problems among sedentary middle. International Journal of Current Advanced Research. 2019. 8: 19185-19188.

6. Trofa DP. The Evidence for Common Nonsurgical Modalities in Sports Medicine, Part 1: Kinesio Tape, Sports Massage Therapy, and Acupuncture. JAAOS Global Research \& Reviews. 2020;4(1): e19.00104.

7. King RB. Stroke Caregivers: Pressing Problems Reported During the First Months of Caregiving. Journal of Neuroscience Nursing. 2010; 42(6).

8. Gupta A. Quality of life and psychological problems in patients undergoing neurological rehabilitation. Annals of Indian zcademy of Neurology. 2008; 11: 225-30.

9. Gainotti G. Relation between depression after stroke, antidepressant therapy, and functional recovery. Journal of neurology, neurosurgery, and psychiatry. 2001; 71(2): 258-261.

10. Odunaiya NA. Attitude and practices of obstetricians and gynecologists towards 
involvement of physiotherapists in management of obstetric and gynecologic conditions. International journal of women's health. 2013; 5:109-114.

11. Shaw IF. Preventive physiotherapy in industry1 1Delivered at the XIV Biennial Congress of the Australian Physiotherapy Association, Sydney, August 1975. Australian Journal of Physiotherapy. 1976; 22(1): 27-31.

12. Padula RS. Physical therapy in occupational health and ergonomics: practical applications and innovative research approaches. Brazilian journal of physical therapy. 2016;20(5): 490-492.
13. Morrison M. Physiotherapy in geriatric rehabilitation. The Journal of the Royal College of General Practitioners. 1969; 18(84 Suppl 1): 3335.

14. Forster A, Lambley R and Young JB. Is physical rehabilitation for older people in long-term care effective? Findings from a systematic review. Age and Ageing. 2010; 39(2): 169-175.

15. Flanagan E, Chopra $\mathrm{T}$ and Mody L. Infection prevention in alternative health care settings. Infectious disease clinics of North America. 2011; 25(1): 271-283. 